\title{
COMPUTATION AND CONSTRUCTION OF VAULT GEOMETRY PROTOTYPES
}

\author{
$\underline{\text { Sambit Datta }}^{1}$, Michael Sharman ${ }^{2}$, Stuart Hanafin $^{2}$, and Teng-Wen Chang ${ }^{3}$ \\ ${ }^{1}$ School of Built Environment, Curtin University, Perth, Australia \\ ${ }^{2}$ School of Architecture and Building, Deakin University, Geelong, Australia \\ ${ }^{3}$ Graduate School of Computational Design, National Yunlin University of Science and Technology (NYUST), \\ Douliou, Taiwan \\ * Corresponding author (sambit.datta@curtin.edu.au)
}

\begin{abstract}
Physical models and scaled prototypes of architecture play an important role in design. They enable architects and designers to investigate the formal, functional, and material attributes of the design. Understanding digital processes of realizing scaled prototypes is a significant problem confronting design practice. This paper reports on three approaches to the translation of Gaussian surface models into scaled physical prototype models. Based on the geometry of Eladio Dieste's Gaussian Vaults, the paper reports on the aspects encountered in the process of digital to physical construction using scaled prototypes. The primary focus of the paper is on computing the design geometry, investigating methods for preparing the geometry for fabrication and physical construction. Three different approaches in the translation from digital to physical models are investigated: rapid prototyping, two-dimensional surface models in paper and structural component models using CNC fabrication. The three approaches identify a body of knowledge in the design and prototyping of Gaussian vaults. Finally the paper discusses the digital to fabrication translation processes with regards to the characteristics, benefits and limitations of the three approaches of prototyping the ruled surface geometry of Gaussian Vaults. The results of each of three fabrication processes allowed for a better understanding of the digital to physical translation process. The use of rapid prototyping permits the production of form models that provide a representation of the physical characteristics such as size, shape and proportion of the Gaussian Vault.
\end{abstract}

Keywords: Parametric Geometry, Digital Fabrication, Physical Prototyping, Gaussian Vault

\section{INTRODUCTION}

Physical models and scaled prototypes of architecture play an important role in design. They enable architects and designers to investigate the formal, functional, and material attributes of the design. Understanding digital processes of realizing scaled prototypes is a significant problem confronting design practice. Using computer-aided modeling functions, the design, representation, and fabrication methods of scaled models can be significantly enhanced $[1,2]$. However, within the digital representation of prototype form, the translation of the geometry to formats suitable for digital fabrication poses considerable challenges for architects and designers.
The paper discusses the possible methods of digital fabrication for scaled prototypes. An integrated design approach, utilizing computer-aided modeling and suitable fabrication processes such as reported by Bechthold [3] are investigated. The forms used for the experimentation in design and fabrication of scaled prototypes were based on Eladio Dieste and his work in masonry vaults and shells [4] Of Dieste's developed forms, the paper focuses on the geometry of the Gaussian vault [5]. The intention of the paper is to understand different processes for translating digital surface geometry of Gaussian Vaults into physical prototypes. The choice of the Gaussian Vault as a prototype was motivated by three qualities of the form: the strict mathematical/structural basis of the form, the presence of 
double curvature on the surface and the possibility to represent the vault using ruled surface geometry.

This was achieved through three separate approaches: solid rapid prototyping, surface model and structural models.

With the surface realised as a digital object, necessary functions can be applied in anticipation for fabrication planning. Furthermore, the capabilities of producing either form within parametric software allowed for the opportunity to apply variable change to the fundamental aspects of size, shape and degree of curvature [1]. Subsequently, the fabrication approaches allow for the physical reformation of the intended prototype form [6]. This methodology lends itself to firstly designing the digital form, evaluating and preparing the required fabrication planning, and finally manufacturing the building members for construction of the physical prototype.

\section{GAUSSIAN VAULT GEOMETRY}

The creation of surface geometry within a digital environment is the initial step to the conception of fabricating the intended form. The surface geometry of the Gaussian Vault is based on two catenary curves of differing heights that sweep across the span of the form. Profiling the progression (perpendicular to the span), the vault starts and ends with straight line profiles.

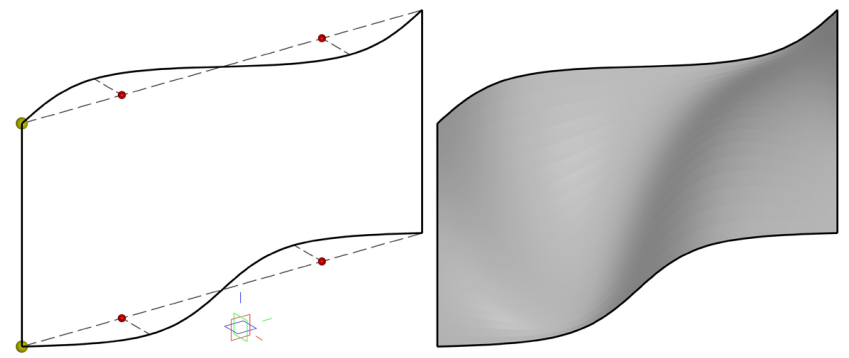

Fig. 1 Development of the Gaussian vault; profile relationships devised by variable base geometry (left), and the eventual development of surface geometry (right).

However, as the profile reaches the apex of the span curves, the shape transforms into an S-curve and creates the degree of double curvature in the form. The surface is an outcome of the development of this geometry (Fig. 1). The digital surface geometry is then translated into scaled prototypes. Three methods are examined using both known and experimental processes based on the forms developed by Dieste $[1,5]$. The first method is through the use of rapid prototyping. This method directly translates the digital model into a physical object. The second is a surface model through unfolding the surface geometry into strips of planar polygons. The third method is through the creation of repeatable component that can apply over a discrete surface. The resulting model can then be prototyped using a $\mathrm{CNC}$ router or similar manufacturing device.

\section{CONSTRUCTION OF PROTOTYPE}

The first phase to understanding the development of scaled prototypes is through rapid prototyping. The process of this methodology is quite simple and not necessarily unique. The surface of the Gaussian vault is thickened by a depth parameter. As a solid digital model, the form is exported into a consistent stereolithography file (.STL) where the solid model is represented as a triangulated mesh. This STL file is then prototyped using a Z450 3D printer (Fig. 2).

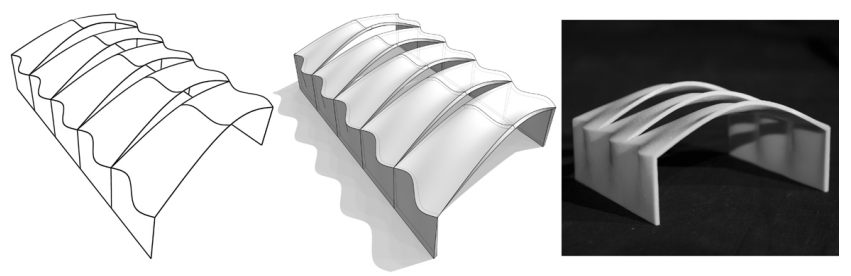

Fig. 2 Rapid prototyping process: surface geometry (left), extrusion to solid (centre), and rapid prototype model (right).

\section{SURFACE MODEL}

The second method involves experimentation with surface geometry using planar strips. The smooth curvature of the Gaussian surface is developed into a planar polygon mesh. To achieve this, the smooth surface is developed using a grid of points applied across the surface geometry. This point grid is then joined together to produce a polygon mesh (Fig. 3). 


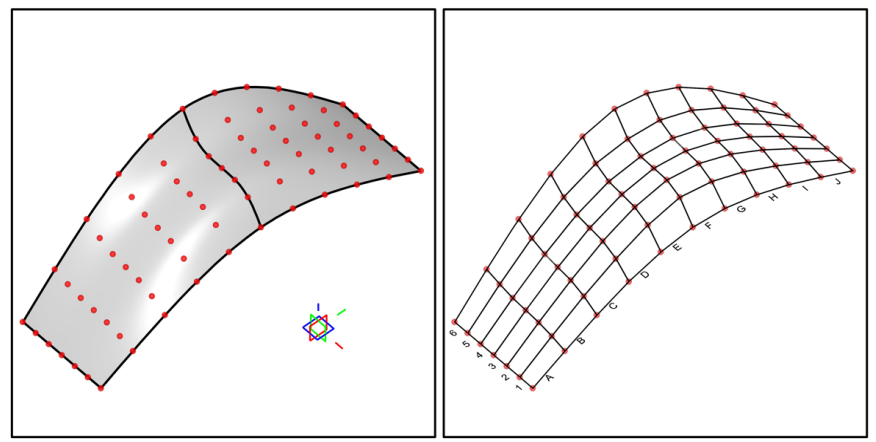

Fig. 3 Preparation of surface geometry for fabrication planning; evaluated surface geometry (left), and discrete polygon mesh (right)

Each band of the polygon mesh is then unfolded into a triangulated planar strip. Two planar strips were developed in the lateral and longitudinal direction of the surface geometry to facilitate an accurate discrete representation of the smooth surface. This was achieved by switching the skin direction of the surface such that paths became profiles and profiles became paths.

The fabrication of the planar strips involve two distinct sets of vector lines; a set of lines that are required to be cut and a set of vector lines that required to be engraved. With the use of a device such as a laser cutter, each set of vector lines (cut or etched) could be distinguished and allow the production of individual planar strips of material. As the surface geometry is to be produced in a set of planar strips, the material constraints are different to the previous method of solid rapid prototyping. Thin card or paper can be used to fold and weave the planar strips together into a three dimensional form (Fig. 4).

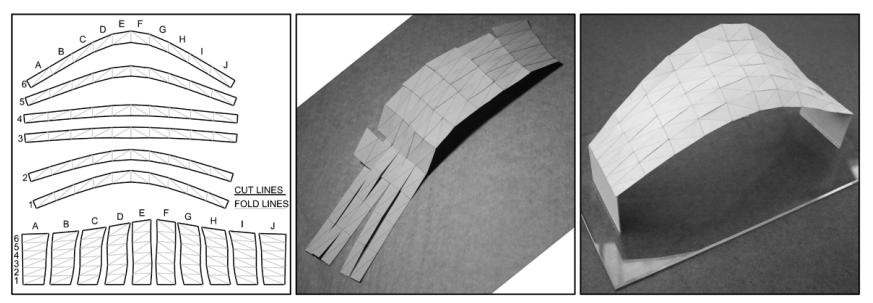

Fig. 4 Stages to unfolding the surface geometry into a physical prototype; fabrication planning of planar strips (left), construction of produced paper strips (centre), and the physical prototype (right).

\section{STRUCTURAL MODEL}

The production of a reticulated prototype involves the design of structural members as developable components. The aim of the prototype is to develop a reticulated grid shell that can be fabricated using planar technology. The Gaussian surface geometry is formulated into a discrete polygon mesh. The mesh is similar to the thin surface method, with the additional constraint that each polygon has to be oriented in strict lateral and longitudinal directions. In other words, the point grid needs to maintain straight directions and not be able to curve away in a third direction.

To be able to constrain the point grid in terms of its lateral and longitudinal bearings, a rectangular projection mesh is created in parallel to curved surface geometry. This mesh produces the required point grid that, once projected in a controlled single direction towards the surface, would constrain the points where they were required (fig. 5).

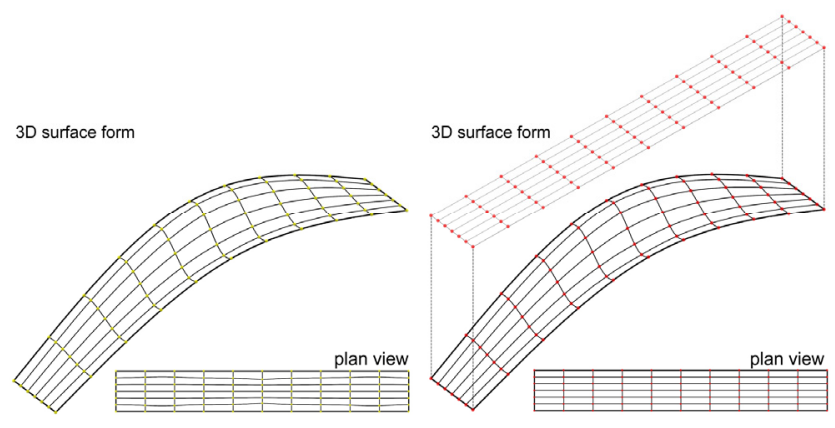

Fig. 5 Development of the Point Grid; the desired distribution is achieved by having the point grid exist in two directions (right) and not in curving with the surface (left).

By utilizing this method of applying the point grid, the points concentrate closer together as the surface form reaches greater curvature. This means that the form is straighter where the points are further apart, thus incurring less structural strain on the member.

The point grid model can be further customized by varying the number of points in the grid. Adding or subtracting points along either lateral or longitudinal directions affects 
the number of structural members used in the prototype model. The use of this variation in the point grid permits the addition and deletion of structural members.

The use of such an evaluated point grid, applied to the surface geometry, allows for the inclusion of a triangulated polygon. The geometric value of the polygon mesh contains relative information such as vertices around each portion of the mesh division, an attribute that would be required to fabricate the structural components.

As the intention of this experiment is to fabricate structural members, depth (i.e. material thickness) is of vital importance to represent the prototype form. Depth is formulated by including an inner and an outer surface geometry developed between two single polygon shapes (quads). The depth of components is then determined by the distance between the meshes; an input that could be controlled within the digital surface form model. The process of assigning thickness is similar to the development of parallel and offset meshes [7].

The construction component development addresses a way to apply solid members between the four vertices points of each of the polygon shapes. Further, the directions used within component have to adhere to individual or combined vertex projections rather than global directions. This meant that the use of a base coordinate system in $\mathrm{X}, \mathrm{Y}$ or $\mathrm{Z}$ directions could not be used.

During the fabrication of the digital component, the control of member sizes, the addition of joinery between crossing members were also explored. The parameters for these explorations were setup through variable change within the component model, which then propagated as inputs to the overall form model (Fig. 6). The primary goal in developing such a constrained structural component is to allow for the automatic evaluation of the Gaussian surface without the need for manual correction on any individual part.

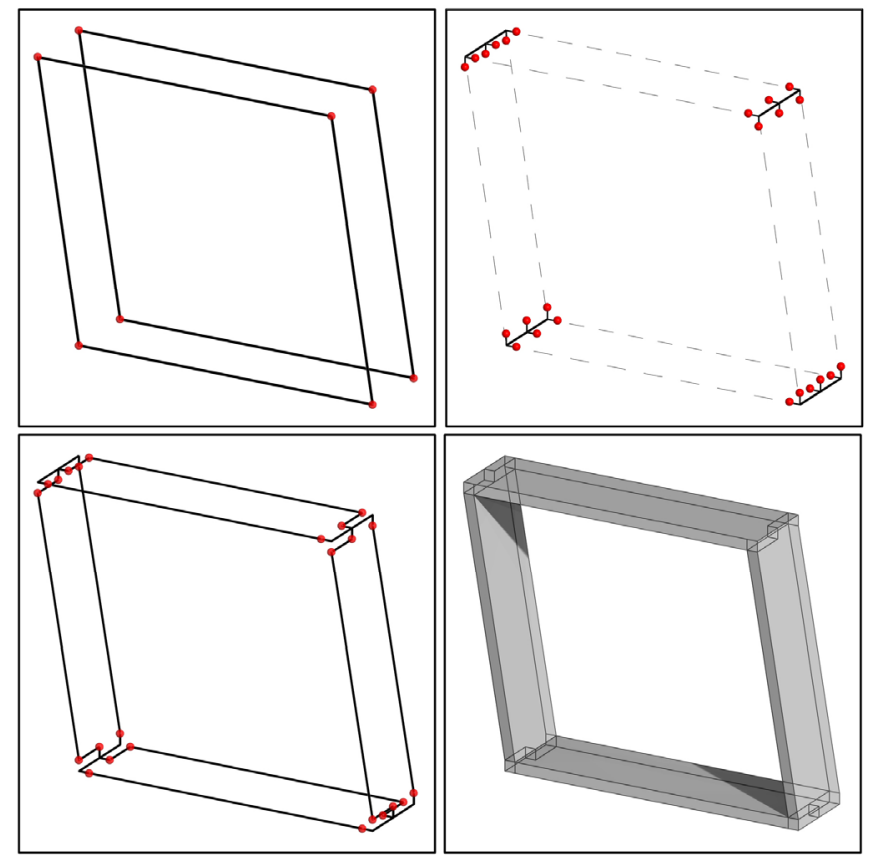

Fig. 6 Development of the Structural Component Model (left), and eventual development (right).

With the component constrained in place over the point grids of the surface geometry, the end result is a simple three dimensional model that can be handled in a standard manner for fabrication. The surface profiles of each of the structural members thus formulated, whether they are exist in the lateral or longitudinal direction, can be extracted for arrangement on a fabrication sheet layout. This information can then be transferred to a planar fabrication device (laser cutter or $\mathrm{CNC}$ cutter) for constructing the structural members. Once the manufacturing is complete, each part is slotted together with the aid of a standard lap joint (Fig. 7). The end result is a physical realization of the desired form as a scaled prototype of the Gaussian Vault as a discrete, reticulated grid shell [8].

\section{RESULTS}

The results of each of three fabrication processes allowed for a better understanding of the digital to physical translation process. The use of rapid prototyping permits the production of form models that provide a representation of the physical characteristics such as size, shape and proportion of the Gaussian Vault. The two processes that develop the surface geometry prototypes through planar fabrication provide a better understanding 
of the skin and structure of the Gaussian Vault. The combination of both prototypes allow for a consideration of the important aspects of the geometry, material and structural characteristics of building envelope [9]. The ability for architects and designers to integrate conceptual form making with structural and material characteristics and its consequent production is enhanced through a better understanding of the processes of digital to physical translation [10]. The scaled prototypes investigated in this paper develop planar fabrication models of complex geometry. For achieving a better integration between the structure and skin, their implications for full-scale modeling and exploration of architectural and structural details, further research into reticulated components [8] and multi-layer meshes [11] is necessary.

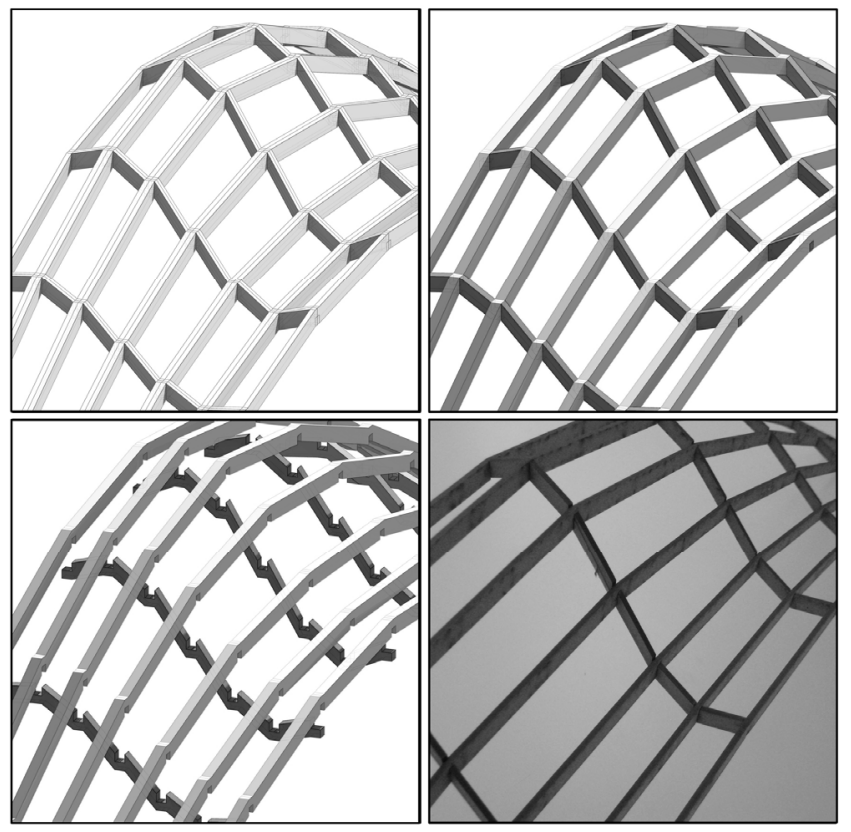

Fig. 7 Development of the Gaussian vault; profile relationships devised by variable base geometry (top left). Development of surface geometry (top right) and Structural fabrication model (bottom, left and right).

\section{REFERENCES}

[1] Aish, R. and Woodbury, R., Multi-level Interaction in Parametric Design Lecture Notes in Computer Science, 3638, 151-162, Springer-Verlag, Berlin, 2005.

[2] Kolarevic, B (ed), Architecture in the Digital Age: Design and Manufacturing, Spon Press, New York, 2003.

[3] Bechthold, M., Surface Structures: Digital Design and Fabrication, ACADIA04, Ontario, pp. 88-99, 2004.

[4] Anderson, S. (ed.): 2004, Eladio Dieste: Innovation in Structural Art, Princeton Architectural Press, New York.

[5] Pedreschi, R.: 2000, The Engineers Contribution to Contemporary Architecture: Eladio Dieste, Thomas Telford, London.

6] Shelden, D.R., Digital Surface Representation and the Constructability of Gehry's Architecture, Ph.D. thesis in Department of Architecture, Massachusetts Institute of Technology, Cambridge, 2002.

[7] Pottman, H., Asperl, A. and Kilian, A. (ed.): 2007, Architectural Geometry, Bentley Institute Press, Exton.

[8] Stephan, S., Sánchez-Alvarez, J., and Knebel, K., Reticulated Structures on Free Form Surfaces, Mero GmbH \& Co., Würzburg, 2005.

[9] Schittich, C. (ed.), in Detail: Building Skins, Birkhäuser, Berlin, 2006.

[10] Glymph, J., Shelden, D., Ceccato, C., Mussel, J. And Schober, H., A parametric strategy for free form glass structures using quadrilateral planar facets. Automation in Construction 13, 187 202, 2004.

[11] Pottmann, H., Liu, Y., Wallner, J., Bobenko, A., and Wang, W., Geometry of multi-layer freeform structures for architecture. ACM Trans. Graphics 26, 3, \#65,1-11, 2007. 